\section{Cahiers de Narratologie}

Analyse et théorie narratives

40 | 2021

École, Récits, Nation

\title{
Contemporary French and Francophone Narratology
}

\section{Arnaud Schmitt}

\section{(2) OpenEdition \\ Journals}

\section{Electronic version}

URL: https://journals.openedition.org/narratologie/12615

DOI: $10.4000 /$ narratologie. 12615

ISSN: $1765-307 X$

Publisher

LIRCES

\section{Electronic reference}

Arnaud Schmitt, "Contemporary French and Francophone Narratology", Cahiers de Narratologie [Online], 40 | 2021, Online since 09 December 2021, connection on 03 April 2022. URL: http:// journals.openedition.org/narratologie/12615 ; DOI: https://doi.org/10.4000/narratologie.12615

This text was automatically generated on 3 April 2022.

Article L.111-1 du Code de la propriété intellectuelle. 


\title{
Contemporary French and Francophone Narratology
}

\author{
Arnaud Schmitt
}

\section{REFERENCES}

ISBN: 9780814214497

1 La narratologie est-elle une affaire de géopolitique ? Et si oui, l'échiquier géopolitique mondial a-t-il évolué définitivement en faveur des États-Unis ? Après avoir été centrale dans les années 70 et 80 , quelle est la place de la France dans la narratologie contemporaine? Qu'en est-il plus largement de la narratologie francophone? Même si ce recueil dirigé par John Pier - figure centrale de la narratologie en France (mais luimême américain) de par ses publications et le séminaire qu'il co-dirige à l'EHESS («Recherches contemporaines en narratologie ») - n'a pas pour finalité de répondre de manière définitive à ces questions, il propose cependant un début de réponse, notamment dans l'introduction rédigée par Pier et dans le dernier article «Policing Literary Theory » dans lequel Françoise Lavocat, autrice de Fait et Fiction (2016), dresse un panorama fascinant de l'évolution de la narratologie et de la théorie littéraire en France et dans le monde ces dernières décennies (l'une des forces de cet ouvrage est notamment de terminer sur ces pages stimulantes). Il est évident pour n'importe quel chercheur plus ou moins impliqué dans l'un des deux plus importants réseaux de narratologie et plus largement de recherche sur le récit, à savoir l'ISSN (International Society for the Study of Narrative) et l'ENN (European Network of Narratology), que la participation française est en général modeste, voire très modeste dans le premier cas. De plus, l'impact d'une revue de narratologie majeure telle que Poétique est dorénavant bien moindre que celui de son pendant américain, Narrative, probablement du fait de la langue dans laquelle sont rédigés les articles qui y sont publiés. Il semble difficile de contredire Françoise Lavocat lorsqu'elle avance que ces dernières décennies en France, peu de chercheurs se réclament de la narratologie ${ }^{1}$. 
2 Jusqu'à un certain point, la France incarne l'ère classique de la narratologie et les ÉtatsUnis, et dans une moindre mesure l'Europe du nord, l'ère post-classique d'une narratologie moins structuraliste et plus ouverte aux influences externes, notamment celles des sciences cognitives et de la théorie de la réception. Mais comme le démontrent John Pier et plus longuement Raphaël Baroni dans le premier article intitulé "Pragmatics in Classical French Narratology and Beyond», cette séparation nette entre ces deux périodes mérite d'être relativisée. Baroni, universitaire suisse qui porte haut et fort les couleurs de la narratologie francophone depuis plusieurs années, se dit convaincu qu'une conception fonctionnaliste du récit, proche donc de la narratologie post-classique, était déjà présente, en tout cas au moins de manière embryonnaire, dans la tradition structuraliste, et donc classique, de la narratologie française ${ }^{2}$. En effet, évoquant la conception assez répandue de l'histoire de la narratologie comme binaire, scindée en modèle A et B (12), le premier ne s'intéressant qu'à ce qui semble être inhérent au texte sans prêter attention à sa dimension pragmatique et communicationnelle et le second se concentrant quant à lui sur l'interaction entre le texte et le travail herméneutique du lecteur ${ }^{3}$, Baroni défend le point de vue selon lequel les ingrédients nécessaires pour développer le modèle $B$ figuraient déjà dans le modèle classique de la fin des années 60 et des années $70^{4}$. Néanmoins, pour expliquer le fait que le modèle B ait connu un essor bien moindre en France qu'outre-Atlantique par exemple, il avance de manière très pertinente l'hypothèse selon laquelle les premiers narratologues français furent réfractaires à évoluer vers le modèle B pour des raisons idéologiques, voire politiques. En effet, des notions purement post-classiques telles que l'intrigue ou la tension narrative dont Baroni est le spécialiste étaient automatiquement associées à l'aspect commercial, 'vendeur' de la littérature ${ }^{5}$, un aspect que les fers de lance du Nouveau Roman par exemple tentaient de neutraliser dans leur fiction.

Remettant en cause lui aussi, en tout cas jusqu'à un certain point, une vision binaire de l'histoire de la narratologie, John Pier décèle également une certaine fluidité dans la circulation des idées, soulignant que les narratologues structuralistes étaient déjà préoccupés par les aspects contextuels du récit ${ }^{6}$. Il est intéressant de noter que Sylvie Patron, une autre figure importante de la narratologie en France qui a récemment présidé l'ISSN, propose une lecture semblable d'un pan plus spécifique des études narratives, à savoir le narrateur (et ses absences) : «We can see that lines of history allow us to make the opposition between optional-narrator theorists and pan-narrator theorists more complex, since opposed theorists do not always occupy opposite lines and since some theorists are not always found on the lines where they would be expected to be » (50). Cette façon de décloisonner l'histoire de la narratologie et de montrer ainsi que la narratologie classique n'était pas que formaliste et envisageait déjà le récit comme expérience représente-t-elle in fine une stratégie de réhabilitation, ou en tout cas de réactualisation de la narratologie française et francophone? Certes, l'importance, voire la nécessité d'avoir une connaissance précise de l'histoire de cette discipline afin d'éviter les malentendus, les références inexactes ou tout simplement les répétitions est soulignée de manière récurrente tout au long de cet ouvrage. Parmi tous les articles, celui rédigé par Sylvie Patron est le texte qui met le plus l'accent sur cette nécessaire rigueur scientifique dont quelques publications récentes semblent manquer. Certaines oppositions théoriques entre chercheurs, bien qu'en principe tout à fait normales et souhaitables pour la diversité du champ, sont quelquefois le fruit d'incompréhensions liées à une connaissance partielle des théories et des concepts 
convoqués. Patron promeut ainsi une recherche qui combine une investigation de la structure des théories et de la genèse des concepts ${ }^{7}$. L'intérêt d'une telle approche est évident puisqu'elle envisage l'histoire non de manière cyclique avec le risque de redite mais bien cumulative (47). Elle démontre dans son article qu'une telle approche, aussi exigeante soit-elle, est possible.

Au-delà de l'approche historique et de la place de la narratologie francophone dans le monde des humanités, on peut bien évidemment se poser la question de la finalité de ce volume, portant certes sur la narratologie française et francophone mais publié en anglais par l'une des maisons d'édition les plus emblématiques de la prépondérance de la narratologie étasunienne, les Presses Universitaires de l'Ohio. Plus qu'un panorama exhaustif de ce qu'est actuellement cette narratologie, les contributions rassemblées dans cet ouvrage représentent surtout ce qu'elle produit de plus stimulant et de plus visible internationalement. Pour autant, il ne faut pas y chercher une unité thématique - à l'exception de la place de la narratologie classique dans l'histoire des études narratives comme évoqué ci-dessus - ou encore des synergies paradigmatiques ou méthodologiques. Si John Pier dans son introduction et dans son article consacré aux possibles vases communicants entre le champ de l'analyse du discours en France et la théorie narrative, Raphaël Baroni, Françoise Lavocat et dans une moindre mesure Sylvie Patron relèvent le défi de l'évaluation de la place de la narratologie francophone (qu'ils incarnent) dans l'histoire de la théorie littéraire, les autres auteurs profitent de ce volume pour évoquer leur propre recherche et continuer à creuser leur sillon en quelque sorte. En conséquence, la lecture de l'ensemble des articles ne manque pas de produire une impression d'hétérogénéité, cependant ces textes témoignent aussi de la diversité et du dynamisme qui caractérisent ce groupe de chercheurs francophones.

Ils démontrent aussi que préoccupations formalistes et influences post-classiques peuvent cohabiter, et qu'il est même incohérent de séparer clairement les deux. Comment peut-on en effet contextualiser un texte sans en considérer la dimension formelle? C'est probablement dans l'article de Claude Calame que cette interdépendance des deux courants est la plus évidente. Dans le sillage de son ouvrage Qu'est-ce que la mythologie grecque ? Calame continue de revisiter les mythes grecs sachant que selon lui le concept même de mythe est inventé par l'anthropologie moderne $^{8}$ - à l'aune de la narratologie. Cette démarche fait totalement sens puisqu'il démontre que la façon dont nous percevons l'histoire de la Grèce antique repose avant tout sur des récits ce qui la rend intrinsèquement narrative. Optant pour une approche sémio-narrative, à la fois structurelle et pragmatique (176), Calame illustre sa démarche en portant son regard analytique sur la quatrième ode pythique de Pindare, et met au jour la façon dont des éléments sémantiques basés sur des représentations culturelles sont imbriqués dans la façon dont le récit est structuré et évolue"

6 Comme évoqué ci-dessus, ce volume est l'occasion pour certain.e.s de revenir sur leurs thèmes de recherche principaux et d'en présenter une synthèse. Sylvie Patron continue à explorer l'opposition entre ceux qui pensent que le texte fictionnel peut occasionnellement ou de manière prolongée se passer de narrateur («non-narrator theory » et "optional-narrator theory ») et ceux qui croient en son ubiquité ("pannarrator theory »), passant en revue avec une précision et une connaissance toujours aussi impressionnantes les arguments des deux camps. À nouveau, nous nous trouvons ici à la jonction des deux grandes périodes de la narratologie puisque le narrateur est logiquement un concept qui a mobilisé les théoriciens de la veine structuraliste et qui 
suscite l'intérêt de la narratologie ultra-contemporaine ${ }^{10}$. Le chercheur québécois Richard Saint-Gelais continue quant à lui à explorer la transfictionnalité sous d'autres angles, et plus spécifiquement ici la façon dont la matérialité du livre a une incidence sur sa lecture, notamment lorsque le récit semble sortir de son cadre pour envahir le paratexte ${ }^{11}$. Il nous montre de manière fascinante comment aucun aspect du paratexte ne peut échapper à une potentielle fictionnalisation, que ce soit par exemple l'épigraphe ou la typographie (le changement de perspective dans le roman Fools de Pat Cadigan étant signalé par un changement de typographie par exemple). Ce que SaintGelais nomme "parafictionalization » (65) fait référence à la façon dont un lecteur traite un élément textuel, matériel donc, en élément diégétique. D'une certaine manière, Benoît Hennaut intègre aussi la matérialité dans son approche théorique, cette fois celle de la représentation scénique, puisqu'il est le spécialiste de la narratologie appliquée au théâtre. Dans son article, il revient sur le problème central de ce type de narratologie : l'absence présumée de narrateur, ou de la source du discours narratif ${ }^{12}$. Hennaut propose de résoudre ce problème par le biais du concept de "super agent désincarné » (« disembodied super-agent», 77), la fonction narrative d'une pièce n'existant qu'à travers sa représentation, sa "performance » (77). Celle-ci est donc à part entière une forme de discours. Il s'intéresse ensuite à une forme de théâtre qu'il nomme " post-dramatique » (" postdramatic performance», 79) et qui semble résister de prime abord à toute forme de narrativité et il revient sur les incarnations narratoriales que ce théâtre peut produire.

7 Toujours dans une logique assez proche de la notion de transfictionnalité, Françoise Revaz étudie «la poétique du récit suspendu " ("The Poetics of Suspended Narrative », 92), plus particulièrement le récit présenté de manière sérielle. La sérialité remet en cause l'unité du texte comme récit, ou du récit comme texte unique, même si les fragments qui structurent le récit sériel se doivent de produire une unité (« to weave a unity that transcends the fragments ", 97). Elle s'intéresse donc plus spécifiquement à ce qui peut produire ce sentiment d'unité narrative indispensable. Il faut en effet une unité thématique (99) pour générer "une totalité émergente» (" an emergent totality ", 100). À nouveau, nous nous trouvons à la frontière de la narratologie classique (les mécanismes de construction du récit) et post-classique (comment la réception de ces mécanismes génère une impression d'unité narrative chez le lecteur). L'article de Denis Bertrand, membre éminent de l'École de Paris, penche plus du côté de la narratologie du $21^{\text {ème }}$ siècle puisqu'il envisage les études narratives en dehors du champ purement littéraire, à l'instar de nombreux ateliers des congrès annuels de l'ISSN. Il s'intéresse à la notion de narrativité et à celle hautement répandue de storytelling (137). Empruntant à Deleuze et Guattari leur concept de "plan d'immanence ", il élabore une réflexion complexe sur la relation entre narratologie et narrativité.

8 Enfin, si ce recueil d'articles est l'occasion de revenir sur l'histoire de la narratologie et ses deux grandes périodes, principalement dans un contexte francophone, l'article d'oliver Caïra permet pour sa part de se projeter vers le futur et d'envisager de nouveaux champs d'application pour cette discipline, même si certains de ces champs comme les jeux vidéo ont déjà été investis par d'éminents chercheurs, Marie-Laure Ryan par exemple. S'appuyant sur une base empirique, ce qu'il appelle « the notebook experiment » (155), consistant à demander à un échantillon de personnes ce qui dans leur expérience récente pouvait être considéré comme fictionnel, Caïra démontre brillamment comment ces expériences fictionnelles vont bien au-delà du périmètre de 
ce qui est étudié actuellement à l'université. Il insiste également sur la nécessité de réunir deux traditions: la fiction mimétique et la fiction logico-mathématique ${ }^{13}$. À l'instar de Benoit Hennaut, il évoque aussi la dichotomie œuvre fictionnelle/ performance fictionnelle et, afin d'intégrer les jeux vidéo au champ des études fictionnelles, envisage ensuite le concept de «fiction non-mimétique» («nonmimetic fiction", 158). Ce que nous comprenons par fiction pourrait alors être représenté par un axe dont les deux pôles opposés seraient le pôle mimétique et le pôle axiomatique ( "mimetic pole » et " axiomatic pole», 160). Il est difficile de ne pas être convaincu par ses arguments selon lesquels «la fictionnalité est une propriété cognitive et pragmatique qui peut être appliquée à n'importe quelle œuvre ou partie de notre expérience $»^{14}$. La narratologie peut donc se préparer à investir de nouveaux champs et ainsi, se renouveler encore une fois.

9 Le fait que Contemporary French and Francophone Narratology soit publié en anglais suggère bien évidemment qu'il n'est pas destiné à un lectorat français - même si un grand nombre de chercheurs francophones en sciences humaines, même en dehors des études anglophones, sont capable de lire en anglais. Le projet initial est donc probablement de remettre cette narratologie francophone non pas au centre mais de le situer sur cet échiquier mondial de la narratologie. Ces dix articles suffiront-ils à atteindre cet objectif? Quelle que soit la réponse à cette question, je suis pour ma part convaincu que ce volume permettra tout simplement de mettre en valeur le travail de dix chercheurs qui ont certes la même langue maternelle mais qui proposent surtout de nouvelles perspectives de recherche pour les narratologues, qu'ils soient francophones ou non. Et en soi, cela constitue déjà un bel accomplissement.

\section{NOTES}

1. "In France, few scholars over the past decades have claimed the title of narratologist » (212).

2. «On looking more closely at my origins, however, I still have the conviction that a functionalist conception of narrative dynamics existed in the French tradition, at least in a latent form, and that my book represents only the tip of an iceberg " (12).

3. "To sum up, Narratologist A is mainly interested in all that belongs objectively to the text, independently of its context and pragmatic considerations, while Narratologist B focuses on the interaction between what is in the text-including reflections on how the author has decided to disclose narrative information-and what the reader can infer during the progressive actualization of the narrative » (16).

4. "The point I want to defend is that almost all ingredients for developing a "B model" of plot dynamics can be found in French narratology, even in the classical modes developed during the late 1960 s and '70s » (18).

5. «In other words, in France, the seductive power of plot, suspense, curiosity, and all the questions revolving around tellability or narrative interests were associated with commercial issues and with outdated, popular, conservative, or right-wing aesthetics, while for all serious intellectuals true art belonged necessarily to the 'writerly' (scriptable) [sic], in other words, the potentially unreadable or unsellable but definitely left-leaning avant-garde » (23). 
6. "Although these questions were somewhat attenuated by French scholars at the time, structural narratology was from the outset open to inquiry into the contextual aspects of narrative that postclassical narratology was later to lay claim to » (2).

7. «In the field of narrative theory, it is important to encourage historical research that combines questions concerning the structure of theories with an examination of the genesis of concepts » (47).

8. "In essence, myth is a concept invented by modern anthropology out of uncertainties gradually recognized by the Greeks themselves as to the historical reality of certain episodes in their own tradition » (1714).

9. "Moreover, recurrence in the text of semantic elements based in cultural representations and embedded in the narrative action and their actors generates figurative 'isotopies,' that is, semantic registers that assure the semantic and logical coherence of narrative and poetic discourse, often in a thread of subtle metaphorical plays » (178).

10. «The narrator is the fundamental concept of classical narratology and has carried on into most postclassical forms of narratology (pan-narrator theories) » (31).

11. "What I would like to examine in this chapter bears more on the opposite situation: cases in which we may ask ourselves whether the narrative is 'spilling over into' the paratext or into elements thereof » (56).

12. "In my opinion, the location of narrative discourse is in fact the primary issue for the narrtological study of theater forms » (75).

13. «So we may want our definition to reunite two long-standing philosophical traditions: fiction based on mimesis and logical-mathematical fiction » (156).

14. «[...] fictionality is a cognitive and pragmatic property that can be applied to any work or segment of experience » (163). 